\title{
Evidence for the existence of two breathinglike phonon modes in infinite bundles of single-walled carbon nanotubes
}

\author{
V. N. Popov* \\ Physics Department, University of Antwerp (RUCA), 2020 Antwerp, Belgium \\ Luc Henrard \\ Laboratoire de Physique du Solide, Facultés Universitaires Notre-Dame de la Paix, 5000 Namur, Belgium
}

(Received 13 November 2000; published 29 May 2001)

\begin{abstract}
We study the low-frequency region of the Raman spectra of infinite bundles of identical single-walled carbon nanotubes within a valence force field model of the lattice dynamics and a bond-polarizability model for the Raman intensity. It is obtained that two breathinglike phonon modes are present in such bundles for each tube type. Based on this prediction, an alternative interpretation of the experimental Raman spectra is proposed. In particular, an explanation is given for the appearence of resonant Raman lines that are predicted as nonresonant within the noninteracting tube picture.
\end{abstract}

DOI: 10.1103/PhysRevB.63.233407

PACS number(s): 63.22.+m, 78.30.-j

Since the discovery of the carbon nanotubes nearly a decade ago, ${ }^{1}$ a significant progress has been achieved in the production of large amounts of single-walled nanotubes (SWNT's). Synthesized by the pulsed laser vaporization process, ${ }^{2}$ by the arc discharge technique, ${ }^{3}$ or by the floating catalyst method, ${ }^{4}$ the SWNT's were found to form triangular lattices (bundles) of up to several hundreds of aligned tubes. Due to their peculiar structure and amazing physical properties, the nanotubes are expected to find many applications. ${ }^{5}$ A crucial step in the nanotube production process is the precise characterization of the nanotube samples. Among the various characterization methods, Raman spectroscopy has proved to provide valuable information for various physical properties of the nanotubes in the samples.

Recently, precise experimental investigations of the Raman scattering of light from phonons in SWNT samples have been carried out. ${ }^{6-12}$ In particular, much attention was given to the study of the low-frequency (LF) region below $500 \mathrm{~cm}^{-1}$ where a high-intensity Raman band is observed. The assignment of this band was carried out using the results of force-constant models of the lattice dynamics of isolated nanotubes, ${ }^{5,13}$ tight-binding ${ }^{14-16}$ and ab initio $^{17-19}$ results for the zone-center phonons, and a bond-polarizability (BP) model $^{13}$ for the Raman intensity. In particular, this Raman band is considered to consist of many Raman peaks, each of which is due to the so-called radial breathing mode (BM) of isolated tubes with given radii and chiral angles. In the BM all carbon atoms experience equal radial displacements. The various models predict that the frequency of the BM is nearly independent of the tube chiral angle and that it is inversely proportional to the tube radius $R$, i.e., it is given by the formula const $R$. The proportionality coefficient was estimated as $1120 \mathrm{~cm}^{-1} \AA{ }^{7,13}$ For the case of infinite bundles of nanotubes, the tight-binding results were fitted with $1307 / R$ for armchair tubes and $1282 / R$ for zigzag tubes. ${ }^{16}$ The $a b$ initio results in Ref. 19 were fitted with [1195 $-25(n-m) / n] / R$ for several tube types $(n, m)$.

It is established experimentally that the LF Raman band is a highly resonant one with peak positions and intensities strongly dependent on the laser line and on the electronic structure of the tubes. ${ }^{19}$ The theory predicts that the Raman intensity of the peaks, due to a given tube type, is enhanced whenever the laser energy becomes equal to the difference between symmetric spikes in the electronic density of states. Moreover, the spikes' separations depend mainly on the tube radius and only slightly on the tube chirality. For practical purposes, these results have been presented in the form of a resonance conditions chart, ${ }^{20}$ allowing one to determine the possible tube types that can contribute to the Raman spectra for a given laser energy. Then, in order to assign the LF Raman band to breathing modes of tubes of certain types, the latter chart is combined with the $1 / R$ dependence of the BM frequency of isolated tubes.

This procedure does not fail in the association of a Raman peak with a metallic or a semiconducting tube in the majority of the cases but in some cases the resonance conditions are fulfilled neither for metallic nor for semiconducting tubes. For example, the observed LF Raman bands for laser lines $E=1.17 \mathrm{eV}, 2.41 \mathrm{eV}, 2.54 \mathrm{eV}$, and $2.71 \mathrm{eV}$ can be attributed to semiconducting tubes and the bands for laser line $E$ $=1.92 \mathrm{eV}$ in can be attributed to metallic tubes. ${ }^{8,9,11}$ The peaks at about $169 \mathrm{~cm}^{-1}$ and $157 \mathrm{~cm}^{-1}$ in the LF band measured at $E=1.59 \mathrm{eV}$ in Ref. 8 may come from metallic tubes. However, the peak at $205 \mathrm{~cm}^{-1}$ in this band does not comply with the resonance conditions and are nonresonant. In the Raman spectra of Ref. 7 taken using excitation energy $E=1.17 \mathrm{eV}$, two subbands are observed in the LF region of the Raman spectra. In one of the subbands, the peaks at $160 \mathrm{~cm}^{-1}$ and $177 \mathrm{~cm}^{-1}$ may originate from semiconducting tubes while the peaks at $230 \mathrm{~cm}^{-1}$ and $252-259 \mathrm{~cm}^{-1}$ in other subband are nonresonant. In two recent papers, ${ }^{10,12}$ some of the samples were found to have wide diameter distributions with tube diameters from 1 to $2 \mathrm{~nm}$ as revealed by the high-resolution transmission electron microscope data. For these samples, the LF Raman band consists of two subbands from 160 to $200 \mathrm{~cm}^{-1}$ and from 220 to $280 \mathrm{~cm}^{-1}$ (see Fig. 4 in Ref. 10) or of a single wide band between 126 and $430 \mathrm{~cm}^{-1}$ (see Fig. 1 in Ref. 12). Although most of the peaks in these spectra can be attributed to resonant scattering 
from metallic and semiconducting tubes, some of the peaks must be nonresonant according to the resonance conditions and the $1 / R$ law. Consequently, the procedure based on the resonance conditions and the $1 / R$ dependence of the BM frequency fails to predict the resonant behavior of certain Raman peaks in several experimental spectra in contradiction with the above-mentioned highly resonant character of the LF Raman band.

Here we provide arguments proving that the mentioned Raman peaks, which the former theory is unable to predict as resonant, can be assigned to Raman-active modes of infinite bundles of identical nanotubes. First, it is shown that in such bundles, two high-intensity Raman peaks are present in the LF region below $500 \mathrm{~cm}^{-1}$. These two peaks are shown to originate from the $\mathrm{BM}$ and a certain double degenerate mode in isolated tubes. The latter mode is of $E_{n}$ symmetry with $n$ depending on the specific tube type and is further denoted as EM (short for $E$ mode). Second, based on these results and the available experimental Raman spectra, we argue that the problematic peaks may be assigned to the higher-frequency mode of both.

The lattice-dynamical study of infinite bundles of identical tubes is accomplished within a valence force field (VFF) model with intertube interactions described by a LennardJones potential. ${ }^{21}$ An infinite bundle is assumed to consist of tubes arranged in a hexagonal crystal lattice for which the lattice parameter is obtained by minimization of the intertube energy. In practice, thorough optimization of the bundle structure must be carried out for tubes in the monoclinic lattice. However, the use of the optimized monoclinic structure is found here to give negligible improvement for the phonon modes over the results for the optimized hexagonal structure and, for this reason and for computational convenience, the latter one is used in the calculations. The Raman spectra are calculated within the BP model ${ }^{13}$ for backscattering geometry and parallel light polarization with averaging over all tube orientations in space.

The results for the Raman spectra for a number of isolated tubes and infinite bundles of tubes of armchair type are shown in Fig. 1. The comparison of the spectra for tubes and for bundles of a given tube type reveals that the BM peak disappears in the latter and, instead of this peak, two other intensive peaks are present that are due to modes denoted here as $\mathrm{BM}(1)$ and $\mathrm{BM}(2)$. For small tube radii, the elastic force constant for radial extension is much larger than the intertube force constant and, consequently, the bundle eigenmodes are nearly unchanged with respect to these of isolated tubes. Then, it is clear from Fig. 2 that $\mathrm{BM}(1)$ originates from the $\mathrm{BM}$ and $\mathrm{BM}(2)$ - from a doubly degenerate mode, $\mathrm{EM}$, in isolated tubes. Both bundle modes are fullysymmetric and can have mixed vibration patterns. It is seen in Fig. 2 that with the increase of the radius, the vibration patterns of the two bundle modes become more and more mixed and similar until they obtain nearly identical breathinglike shapes (apart from a rotation at $\pi / 6$ around the tube axis) for bundles of tubes larger than $(15,15)$, i.e., $R$ $\gtrsim 10 \AA$. The strong mode mixing for large radii is due to fact that the BM and the EM have close frequencies and that the intertube force constant is much larger than the elastic

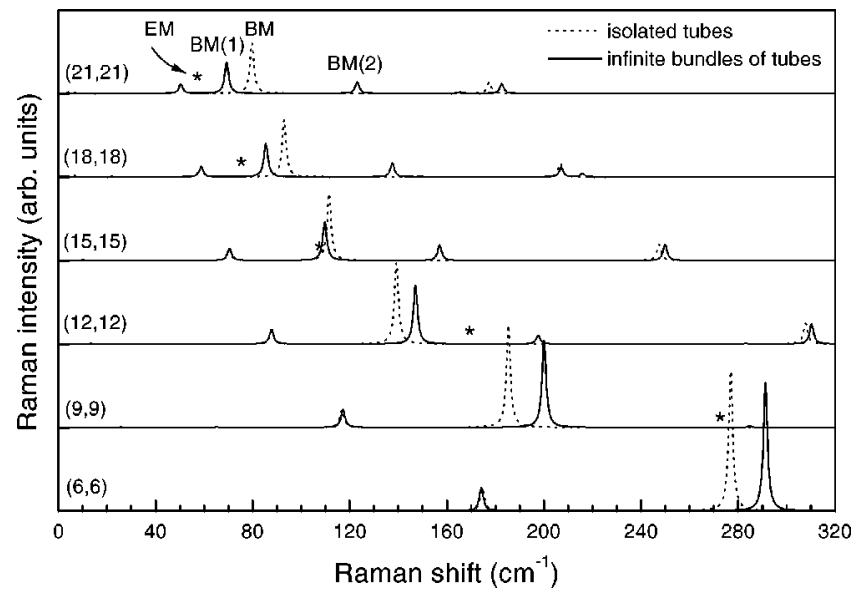

FIG. 1. The Raman spectra of isolated tubes (dotted lines) and infinite bundles of tubes (solid lines) of the armchair types $(6,6)$, $(9,9),(12,12),(15,15),(18,18)$, and $(21,21)$ with radii in the interval from $4 \AA$ to $16 \AA$. The peaks originating from the $\mathrm{BM}, \mathrm{BM}(1)$, and $\operatorname{BM}(2)$ are indicated by corresponding symbols. The asterisk indicates the frequency of the Raman-inactive EM.

one. The radius dependence of the calculated frequencies of both modes is illustrated in Fig. 3 for all armchair and zigzag tube types with radii from $4 \AA$ to $16 \AA$. The calculated Raman intensity of these modes versus tube radius is shown in the inset of Fig. 3, from which it can be concluded that the relative intensity of $\mathrm{BM}(2)$ with respect to $\mathrm{BM}(1)$ reaches a
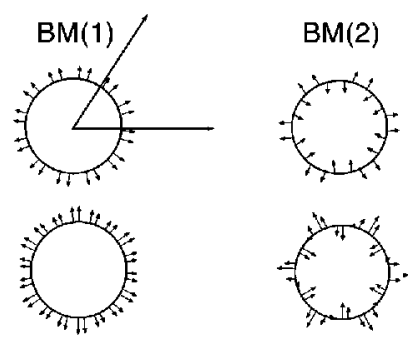

$(12,12)$<smiles>C1=CC=CC=CCC=CC=C1</smiles><smiles>c1ccccc1</smiles>

$(15,15)$<smiles>c1ccccc1</smiles><smiles>c1ccccc1</smiles>

$(18,18)$<smiles>C1=CC=CCC=C1</smiles>

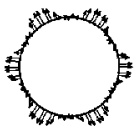

$(21,21)$<smiles>c1ccccc1</smiles>

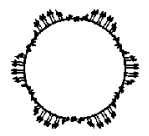

FIG. 2. The atomic displacement patterns for the two breathinglike modes, $\mathrm{BM}(1)$ and $\mathrm{BM}(2)$, in infinite bundles of armchair nanotubes of the types $(6,6),(9,9),(12,12),(15,15),(18,18)$, and $(21,21)$. The circular cross sections of the tubes (scaled to have equal radii) and the primitive translation vectors of the regular triangular lattice are shown. The atomic displacements of the atoms are depicted by arrows. 


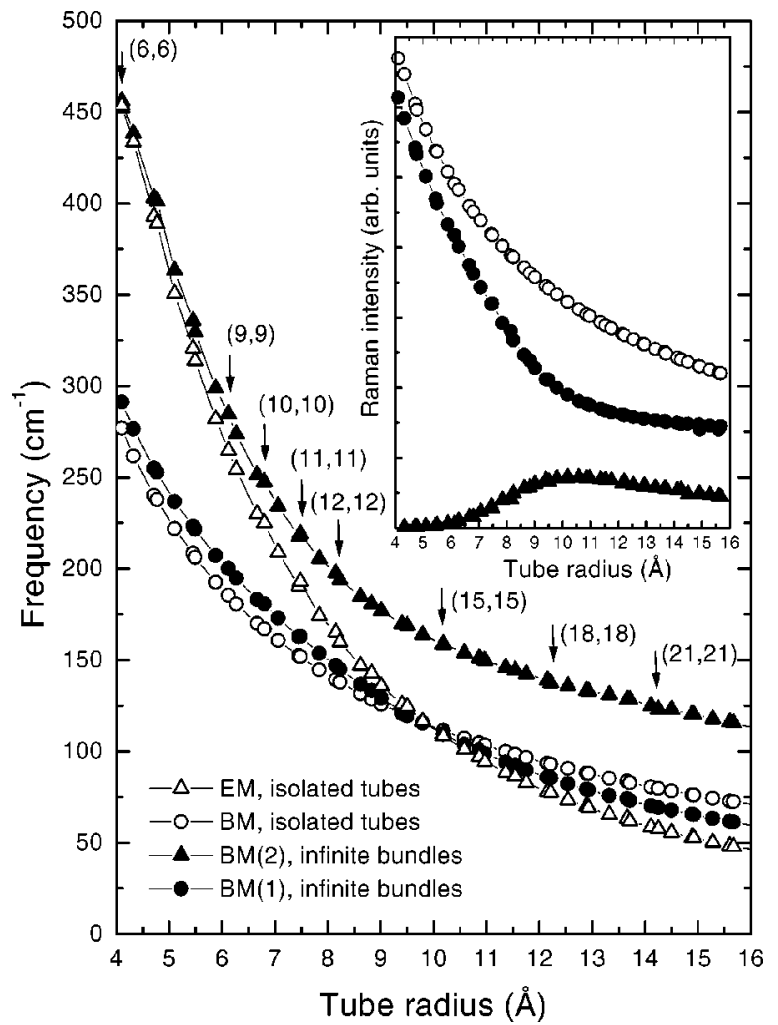

FIG. 3. The calculated frequencies of the BM and the EM in isolated nanotubes and the originating from them $\mathrm{BM}(1)$ and $\mathrm{BM}(2)$ in infinite bundles of identical nanotubes for all armchair and zigzag types with radii between $4 \AA$ and $16 \AA$, i.e., tube types from $(6,6)$ to $(21,21)$ and from $(11,0)$ to $(40,0)$. The inset shows the calculated intensities of these modes vs tube radius.

maximum of about 0.4 for $R \approx 10 \AA$ and decreases to 0.3 for $R \approx 16 \AA$.

In previous calculations of the vibrations of nanotube bundles, a second breathinglike mode has not been obtained because of the limited diameter range of interest ${ }^{22}$ or because of the considered rigid tube breathing only. ${ }^{16}$ However, very recent tight-binding molecular-dynamics calculations of several tube types with radii in the interval $3.5<R<9.5 \AA$ have revealed the existence of the second breathinglike mode in infinite bundles. ${ }^{23}$ The estimated ratio $I_{2} / I_{1}$ of the Raman intensities of the $\mathrm{BM}(2)$ and $\mathrm{BM}(1)$ peaks, based only on the eigenvectors of the BM and the EM, was found to increase with the tube radius and reach the value of about 0.9 for $R$ $\approx 9.5 \AA$ while here we obtain $I_{2} / I_{1} \approx 0.3$.

We apply the obtained results to the assignment of the Raman peaks in the LF part of several experimental spectra. For this purpose, we use the resonance conditions chart and the $1 / R$ law to analyze the resonance behavior of the LF features in these spectra. In the case of several high-intensity Raman lines, this procedure predicts off-resonant scattering. We group each of these lines with a lower-frequency one that is resonant according to the resonance condition and the $1 / R$ law. Further on, we suppose that each such pair of peaks comes from a couple of modes $\mathrm{BM}(1)$ and $\mathrm{BM}(2)$ in infinite bundles of identical tubes. For this to be true for a given pair of peaks, it is necessary that the corresponding radii $R_{1}\left[R_{2}\right]$ obtained from the curves for the lower-frequency mode $\mathrm{BM}(1)$ [higher-frequency mode $\mathrm{BM}(2)$ ] in Fig. 3 were close to each other. The results for such pairs of peaks with close $R_{1}$ and $R_{2}$ are shown in Table I. In the first three columns of Table I, the laser excitation lines used in the Raman scattering experiments and the pairs of observed peaks positions with $\left|R_{1}-R_{2}\right| \leqq 0.3 \AA$ are displayed together with the pairs of radii $\left(R_{1}, R_{2}\right)$. All nanotube types with radii in the $\pm 0.15 \AA$ interval from the average value $\left(R_{1}+R_{2}\right) / 2$ that could be responsible for the pairs of Raman peaks are given in the last column of Table I. It is thus clear that each of the pairs of peaks may originate from one of the several listed tube types but it is not possible to point out a unique tube type due to the assumptions made in these calculations. In

TABLE I. Assignment of several pairs of observed Raman peaks to $\mathrm{BM}(1)$ and $\mathrm{BM}(2)$ of infinite bundles of identical tubes. The first three columns contain the laser excitation energy used to obtain a given Raman spectrum, pairs of observed peak positions in the spectrum (for which $\left|R_{1}-R_{2}\right|<0.3 \AA$ with $R_{1}$ and $R_{2}$ derived from Fig. 3), and the corresponding pairs of radii $\left(R_{1}, R_{2}\right)$. All tube types with radii in the interval $\pm 0.15 \AA$ from the average value $\left(R_{1}+R_{2}\right) / 2$ that could give rise to the pairs of Raman peaks are shown in the last column in order of increasing tube radius.

\begin{tabular}{cccc}
\hline \hline $\begin{array}{c}\text { Excit. energy } \\
(\mathrm{eV})\end{array}$ & $\begin{array}{c}\text { Pair of peaks pos. } \\
\left(\mathrm{cm}^{-1}\right)\end{array}$ & $\begin{array}{c}\left(R_{1}, R_{2}\right) \\
(\AA)\end{array}$ & $\begin{array}{c}\text { Types } \\
\text { of tubes }\end{array}$ \\
\hline $1.17^{\mathrm{a}}$ & $(160,230)$ & $(7.58,7.19)$ & $(14,7),(18,1),(17,3),(19,0),(16,5),(12,10),(18,2)$, \\
& & $(13,9)$ \\
$1.17^{\mathrm{a}}$ & $(177,252)$ & $(6.92,6.70)$ & $(17,0),(16,2),(11,9),(15,4),(12,8),(17,1)$ \\
$1.59^{\mathrm{b}}$ & $(157,205)$ & $(7.65,7.90)$ & $(19,1),(18,3),(13,10),(17,5)$ \\
$1.92^{\mathrm{c}}$ & $(167,240)$ & $(7.25,6.94)$ & $(15,5),(11,10),(13,8),(14,7),(18,1)$ \\
$1.92^{\mathrm{c}}$ & $(189,258)$ & $(6.43,6.52)$ & $(11,8),(16,1),(15,3),(14,5)$ \\
$1.92^{\mathrm{d}}$ & $(157,215)$ & $(7.65,7.55)$ & $(12,10),(18,2),(13,9),(17,4),(15,7),(16,6)$ \\
$1.92^{\mathrm{d}}$ & $(189,254)$ & $(6.43,6.64)$ & $(11,8),(16,1),(15,3),(14,5)$ \\
\hline \hline
\end{tabular}

${ }^{\mathrm{a}}$ Reference 7.

${ }^{\mathrm{b}}$ Reference 8.

${ }^{\mathrm{c}}$ Reference 10 .

${ }^{\mathrm{d}}$ Reference 12 . 
fact, the pair of peaks is supposed to originate from an infinite bundle while a real bundle consists of hundreds of tubes of similar radii. ${ }^{24}$ Consequently, the real peak positions may deviate from the predicted ones here by a few $\mathrm{cm}^{-1}$ depending on the production method of the nanotube samples. Apart from this, the observed peak positions may be shifted with respect to the phonon frequencies due to the resonant character of the light scattering. In metallic tubes, the phonon frequencies themselves can be modified because of electronphonon interactions that are not accounted for in the present calculations.

Note that it is also possible to group some other experimental peaks in pairs for which both peaks could be assigned to resonant scattering involving the BM modes of two tube types. However, even in such cases the possibility of an interpretation in terms of $\mathrm{BM}(1)$ and $\mathrm{BM}(2)$ modes of a single tube type should not be ruled out. If both assignments are plausible, a more refined theoretical approach has to be used in order to distinguish between the two cases.

In conclusion, we have studied the low-frequency region of the Raman spectra of infinite bundles of SWNT's within a VFF model of the lattice dynamics and a BP model for the Raman intensity. The results of the calculations show that in this region there are two breathinglike modes that may give rise to Raman peaks of high intensity. The frequencies and the vibration patterns of these two modes are modified by the intertube interactions especially for tube radii larger than $10 \AA$ where the intertube forces prevail over the elastic forces of the tubes. Since the resonance conditions are practically the same for both modes, it can be expected that the ratio of their Raman intensities, calculated here, will be in semi-quantitative correspondence with the experimental one. Based on these results, we explain the appearance in several experimental Raman spectra of resonantly enhanced Raman peaks that are otherwise predicted to be nonresonant according to the noninteracting tube picture and the resonance conditions.

V.N.P. acknowledges the financial support by Grant No. G.0347.97 of the Flemish Science Foundation, by the Concerted Action of the University of Antwerp GOA-BOF-UA, and by Grants No. CRG 973953 and No. PST.CNS.976098 from the Scientific Affairs Division of NATO. L.H. thanks $\mathrm{Ph}$. Lambin for stimulating discussions. L.H. receives support from the Belgian National Fund (FNRS). This work was partly funded by the Belgian Interuniversity Research Project on Reduced Dimensionality Systems (PAI-UAP P4-10).
*Permanent address: Faculty of Physics, University of Sofia, BG1164 Sofia, Bulgaria.

${ }^{1}$ S. Iijima, Nature (London) 354, 56 (1991); S. Iijima and T. Ichihashi, ibid. 363, 603 (1993); D.S. Bethune, C.-H. Kiang, M.S. de Vries, G. Gorman, R. Savoy, J. Vasquez, and R. Beyers, ibid. 363, 605 (1993).

${ }^{2}$ A. Thess, R. Lee, P. Nikolaev, H. Dai, P. Petit, J. Robert, C. Xu, Y.H. Lee, S.G. Kim, A.G. Rinzler, D.T. Colbert, G.E. Scuseria, D. Tománek, J.E. Fischer, and R.E. Smalley, Science 273, 483 (1996).

${ }^{3}$ C. Journet, W.K. Maser, P. Bernier, A. Loiseau, M. Lamy de la Chapelle, S. Lefrant, P. Deniard, R. Lee, and J.E. Fischer, Nature (London) 388, 756 (1997).

${ }^{4}$ H.-M. Cheng, F. Li, and M.S. Dresselhaus, Appl. Phys. Lett. 72, 3282 (1998).

${ }^{5}$ M.S. Dresselhaus, G. Dresselhaus, and P.C. Eklund, Science of Fullerenes and Carbon Nanotubes (Academic, New York, 1996).

${ }^{6}$ A.M. Rao, E. Richter, S. Bandow, B. Chase, P.C. Eklund, K.A. Williams, S. Fang, K.R. Subbaswamy, M. Menon, A. Thess, R.E. Smalley, G. Dresselhaus, and M.S. Dresselhaus, Science 275, 187 (1997).

${ }^{7}$ S. Bandow, S. Asaka, Y. Saito, A.M. Rao, L. Grigorian, E. Richter, and P.C. Eklund, Phys. Rev. Lett. 80, 3779 (1998).

${ }^{8}$ M.A. Pimenta, A. Marucci, S.D.M. Brown, M.J. Matthews, A.M. Rao, P.C. Eklund, R.E. Smalley, G. Dresselhaus, and M.S. Dresselhaus, J. Mater. Res. 13, 2396 (1998).

${ }^{9}$ G.S. Duesberg, W.J. Blau, H.J. Byrne, J. Muster, M. Burghard, and S. Roth, Chem. Phys. Lett. 310, 8 (1999).
${ }^{10}$ L. Alvarez, A. Righi, T. Guillard, S. Rols, E. Anglaret, D. Laplaze, and J.-L. Sauvajol, Chem. Phys. Lett. 316, 186 (2000).

${ }^{11}$ M.N. Iliev, A.P. Litvinchuk, S. Arepalli, P. Nikolaev, and C.D. Scott, Chem. Phys. Lett. 316, 217 (2000).

${ }^{12}$ P.-H. Tan, Y. Tang, C.-Y. Hu, F. Li, Y.-L. Wei, and H.-M. Cheng, Phys. Rev. B 62, 5186 (2000).

${ }^{13}$ R. Saito, T. Takeya, T. Kimura, G. Dresselhaus, and M.S. Dresselhaus, Phys. Rev. B 57, 4145 (1998).

${ }^{14}$ J. Yu, R.K. Kalia, and P. Vashishta, J. Chem. Phys. 103, 6697 (1995).

${ }^{15}$ D. Kahn and J.-P. Lu, Phys. Rev. B 60, 6535 (1999).

${ }^{16}$ L. Henrard, E. Hernández, P. Bernier, and A. Rubio, Phys. Rev. B 60, R8521 (1999).

${ }^{17}$ J. Kürti, G. Kresse, and H. Kuzmany, Phys. Rev. B 58, R8869 (1998).

${ }^{18}$ D. Sánchez-Portal, E. Artacho, and J.M. Soler, Phys. Rev. B 59, 12678 (1999).

${ }^{19}$ M. Milnera, J. Kürti, M. Hulman, and H. Kuzmany, Phys. Rev. B 84, 1324 (2000).

${ }^{20}$ H. Kataura, Y. Kumazawa, Y. Maniwa, I. Umezu, S. Suzuki, Y. Ohtsuka, and Y. Achiba, Synth. Met. 103, 2555 (1999).

${ }^{21}$ V.N. Popov, V.E. Van Doren, and M. Balkanski, Solid State Commun. 114, 395 (2000).

${ }^{22}$ U.D. Venkateswaran, A.M. Rao, E. Richter, M. Menon, A. Rinzler, R.E. Smalley, and P.C. Eklund, Phys. Rev. B 59, 10928 (1999).

${ }^{23}$ M.S. Desselhaus and P.C. Eklund, Adv. Phys. 49, 705 (2000).

${ }^{24}$ L. Henrard, A. Loiseau, C. Journet, and P. Bernier, Eur. Phys. J. B 13, 661 (2000). 